\title{
Assessment of orofacial functions in Brazilian children using the Nordic Orofacial Test-Screening (NOT-S)
}

\author{
Avaliação das funções orais em crianças brasileiras por \\ meio do Nordic Orofacial Test-Screening (NOT-S)
}

\begin{abstract}
Purpose: Orofacial function is the result of complex activities of the central nervous and neuromuscular systems. Orofacial dysfunction can compromise vital actions, such as breathing, chewing and swallowing, and facial expressions. The aim of this study was to apply the Brazilian version of the Nordic Orofacial Test-Screening (NOT-S), which assesses orofacial dysfunction, to a sample of Brazilian children and adolescents.

Methods: The Brazilian version of NOT-S was applied in 197 girls and 135 boys, aged from 8 to 14 years old. They were clinically examined for the phase of dentition and characteristics of occlusion. For assessing NOT-S reliability, a test-retest was performed in 50 subjects randomly selected from the total sample 15 days after the first application. Descriptive statistics, Pearson's chi-squared, Mann-Whitney and Kappa tests were applied for data analysis at significant level of $\alpha=0.05$

Results: The scores ranged from 0 to 7 ; score 0 had a rate of $5 \%$; the mean score was 2.64 . The most frequent domains were III (Habits) and IV (Chewing and Swallowing) with a rate of 70 and $50 \%$, respectively. No difference between genders was seen in relation to orofacial dysfunction, but subjects in mixed dentition and those with frontal open bite presented more orofacial dysfunction. The rate of intra-examiner agreement was $97.8 \%$ comparing the first and retest applications.

Conclusion: No differences between genders were detected, but subjects in mixed dentition and in those with anterior malocclusion the orofacial dysfunction was more present. The Brazilian version of NOT-S was considered proper for application in Brazilian subjects.
\end{abstract}

Key words: Child; disability evaluation; reproducibility of results; validation studies

\section{Resumo}

Objetivo: A função orofacial é resultado de atividades complexas integradas ao Sistema Nervoso Central e ao Sistema Neuromuscular. A disfunção orofacial pode comprometer ações vitais, como a respiração, a mastigação, a deglutição e as expressões faciais. O objetivo deste estudo foi traduzir o protocolo do Nordic Orofacial Test-Screening (NOT-S), que avalia disfunção orofacial, para o Português do Brasil e aplicá-lo à uma amostra de crianças e adolescentes (8 a 14 anos).

Metodologia: A versão brasileira do NOT-S foi aplicada em 197 meninas e 135 meninos na faixa etária de 8 a 14 anos. Estes foram examinados clinicamente considerando as fases da dentição e características da oclusão. Para avaliar a reprodutibilidade do NOT-S foi aplicado o teste-reteste em 50 indivíduos aleatoriamente selecionados da amostra 15 dias após a primeira avaliação. Estatística descritiva, qui-quadrado, os testes Mann-Whitney e Kappa foram aplicados para análise dos dados, com nível de significância de $\alpha=0.05$.

Resultados: Os escores variaram de 0 a 7 ; o escore 0 apresentou frequência de $5 \%$ e o escore médio foi 2,64. Os domínios III (Hábitos) e IV (Mastigação e Deglutição) foram os mais frequentes e apresentaram taxa de 70 e $50 \%$, respectivamente. Não houve diferença entre os gêneros em relação à disfunção orofacial, porém os escores do NOT-S foram maiores na dentição mista quando comparada à dentição permanente. A taxa de concordância intraexaminador foi de $97,8 \%$ comparando a primeira aplicação com a do re-teste.

Conclusão: Não houve diferença entre gêneros, mas nos indivíduos na dentição mista e aqueles com mordida aberta anterior a disfunção orofacial foi mais presente. A versão brasileira do NOT-S foi considerada adequada para aplicação em indivíduos brasileiros.

Palavras-chave: Criança; avaliação da deficiência; reprodutibilidade dos testes; estudos de validação

\author{
Marina Severi Leme a \\ Taís de Souza Barbosa a \\ Maria Beatriz Duarte Gaviáo o
} a Department of Pediatric Dentistry, Pedodontic
Division, Piracicaba Dental School, State University
of Campinas - UNICAMP, Piracicaba, SP, Brazil

\section{Correspondence:}

Maria Beatriz Duarte Gavião

Faculdade de Odontologia de Piracicaba/UNICAMP Departamento de Odontologia Infantil - Área de Odontopediatria

Avenida Limeira 901

Piracicaba, SP - Brazil

$13414-903$

E-mail: mbgaviao@fop.unicamp.br

Received: February 21, 2012

Accepted: March 21, 2012

Conflict of Interests: The authors state that there are no financial and personal conflicts of interest that could have inappropriately influenced their work.

Copyright: (c) 2011 Leme et al.; licensee EDIPUCRS. This is an Open Access article distributed under the terms of the Creative Commons AttributionNoncommercial-No Derivative Works 3.0 Unported License. 


\section{Introduction}

Orofacial function includes vital actions (e.g., breathing, chewing and swallowing) (1), muscle posture (e.g., mouth and tongue posture) $(2,3)$ that are the basis for social interactions in terms of speech, emotional communication, facial expression and appearance (1). Orofacial function is the result of complex activities of the central nervous system and the neuromuscular system (4). Orofacial dysfunction is a common feature in many genetic and congenital disorders. However, it may also be acquired as a consequence of diseases or trauma (1), and includes the presence of various habits (3).

Due to the key roles of orofacial function, the Nordic Orofacial Test-Screening (NOT-S) was developed and validated to asses orofacial function in all individuals more or equal than 3 years old. It can be performed quickly, without use of special equipment, and is easily used by professionals with different backgrounds. NOT-S was developed by a working group formed at the Second Nordic Conference on Orofacial Therapy in Gothenburg, 2002 and consists of a structured interview and a clinical examination, totalizing 12 domains. Each domain assesses one orofacial function (1).

Globalization of patient care and medical research requires international instruments to assess health status. However, such instruments are usually developed in a specific cultural environment, and a process of cultural adaptation is needed to transfer the protocol to a different culture $(5,6)$. The NOT-S is available in several languages, such as, Danish, English, Finnish, Icelandic, Norwegian and Swedish and recently a Brazilian version was developed by Leme et al. (7). As such, the purpose of this study was to apply the Brazilian version of NOT-S in Brazilian children and adolescents and, consequently, validate this version, in order to make its use possible in any part of the country.

\section{Methods}

The research project was submitted to and approved by the Research Ethics Committee (No.009/2008) of the Piracicaba Dental School - University of Campinas. The consent of the children and their parents/guardians was obtained.

NOT-S consists of a structured interview and a clinical examination, and each part has six sections (domains). Functions assessed in the interview are: I-Sensory Function, II-Breathing, III-Habits, IV-Chewing and Swallowing, V-Drooling and VI-Dryness of the mouth. Functions assessed in the clinical examination are: 1-Face at Rest, 2-Nose Breathing, 3-Facial Expression, 4-Masticatory Muscle and Jaw Function, 5-Oral Motor Function and 6-Speech. Each domain contains one to five items, reflecting the complexity of the specific function. If the answer to one of the questions or the performance on one of the tasks met the criterion for impaired function, the item was recorded as "yes", indicating a dysfunction in the respective domain (1).
The Brazilian version of NOT-S was obtained by a screening process for cross-cultural adaptation. For more details, please see Leme et al. (7). This process is briefly described below.

The English version of NOT-S was initially translated into Brazilian Portuguese language by a dentist with fluency in both English and Portuguese languages (Brazilian version $\mathrm{n}^{\mathrm{o}} 1$ ). This version was next back-translated to English by other dentist, who was native English speaker. Both versions were compared by the two translators, and sentences that seemed different were rewritten until a consensus had been reached, obtaining the Brazilian version $\mathrm{n}^{\circ} 2$. This second version was evaluated by a review committee, formed by three dentist researchers, a formally instructed patient and the first author (MSL), who substituted technical terms by culturally accepted ones, when indicated, obtaining the Brazilian version $\mathrm{n}^{\circ} 3$. The cultural equivalence of the third version was verified by its application in 20 subjects, 8 to 14 years old, of both genders. Alternative answers "I did not understand" and "not applied" were added to each question of NOT-s for identifying the questions and examination commands that were not understood by the subject. The ones that were chosen by $15 \%$ or more subjects were discussed by the committee, who replaced problematic items with culturally accepted ones (8). Next, the protocol was applied to other groups $(n=20)$, until the alternative answer has not been chosen for any question by $85 \%$ or more subjects. Thus, the protocol was considered culturally equivalent and ready for application on a larger sample (7).

\section{NOT-S application}

NOT-S was applied to 332 children who were 8 to 14 years old (mean age: 10.48 \pm 1.69 years; 197 girls and 135 boys, representing $59.45 \%$ and $40.54 \%$ respectively), who did not have systemic and/or mental developmental disorders and were recruited from four public schools in Piracicaba. These children were clinically examined for the phase of dentition and characteristics of occlusion by two examiners, who were previously calibrated (inter-examiner kappa score: 0.88; and intra-examiner kappa score: 0.97), using the evaluation form from World Health Organization (WHO) (9).

In relation to dentition, the sample was subdivided in two groups: Mixed Dentition and Permanent Dentition with 198 $(59.45 \%)$ and 134 (40.54\%) subjects, respectively. Children in the Mixed Dentition were all in the second transitory period. The Mixed Dentition group had a mean age of $9.57 \pm 1.21$, years and the permanent dentition group had a mean age of $11.82 \pm 1.38$ years.

In accordance with the characteristics of occlusion, the sample was classified into normal occlusion $(\mathrm{n}=118)$ and malocclusion $(\mathrm{n}=214)$ groups. Malocclusion included Increased overjet $(\mathrm{n}=123)$, Posterior crossbite $(\mathrm{n}=50)$, Frontal open bite $(\mathrm{n}=24)$, Deep overbite $(\mathrm{n}=67)$, Angle Class II ( $\mathrm{n}=81)$, Angle Class III ( $\mathrm{n}=24)$. Gender, phase of dentition and characteristics of occlusion were considered to evaluate discriminant validity. 
NOT-S was applied individually by the same researcher on a vacant class, just prior to the clinical examination. The NOT-S interview was conducted by reading the questions written in the manual. To assess orofacial dysfunction upon clinical examination, the subjects were requested to perform tasks for each item. The examination was completed in conjunction with an illustrated manual (http://mun-h-center. se/sv/Mun-H-Center), and each sentence had criteria for impaired function. Each YES answer in a section, both in interview and clinical examination, was given a score of 1 , indicating a dysfunction in the scored domain. Each NO answer was given a score of 0 . The total score was the sum of the scores of each section, and ranged from 0 to 12 . On asking the questions of domain III of NOT-S (Habits) the type of habits related by subjects were marked on NOT-S paper.

\section{Reliability}

The reproducibility (test-retest reliability) of the Portuguese (Brazilian) version of NOT-S was assessed 15 days after the first application, by the reapplication of the protocol (test-retest), by the same researcher, in 50 subjects randomly selected from the total sample $(n=332)$ that participated in the first test.

\section{Statistical analysis}

Collection, evaluation and statistical analysis of the data was conducted using Microsoft Office Excel (Microsoft for Windows 2007, USA), Bioestat Version 5.0 (Instituto de
Mamirauá. Belém, PA - Brazil) and SPSS (for Windows 9.0, Chicago, IL - USA). Descriptive evaluation of quantitative parameters was performed by calculating means and standard deviations; qualitative parameters were described by evaluating frequency and percentage rates. Pearson's chisquared test was used for the comparison of percentages, and the Mann-Whitney test was used for comparison of means between groups. All p-values result from bilateral tests, and $\mathrm{p} \leq 0.05$ was defined as significant. Test-retest reliability was assessed by the percentage of intra-examiner agreement and the Kappa value. The discriminant validity was assessed by comparing NOT-S scores between genders, mixed and permanent dentition groups and normal occlusion with different malocclusions groups, using the Mann-Whitney test.

\section{Results}

\section{NOT-S application}

The application time of NOT-S ranged from 5 to $7 \mathrm{~min}$. All subjects answered the interview questions without parental/guardian assistance, based on a pilot test conducted by this research group that verified that children older than 7 years old are capable of answer the interview questions by themselves. For the total sample $(n=332)$, the NOT-S scores ranged from 0 to 7 . A score of 3 was the most frequent, followed by 2,1 and 4 . Scores of 0 were recorded with a frequency of just $5 \%$. The frequencies of the scores according to each group are shown in Table 1.

Table 1. Distribution and frequency (\%) of NOT-S scores for the total sample

\begin{tabular}{|c|c|c|c|c|c|c|c|c|c|}
\hline & & \multicolumn{8}{|c|}{ NOT-S score } \\
\hline & & 0 & 1 & 2 & 3 & 4 & 5 & 6 & 7 \\
\hline Total & & $\begin{array}{c}17 \\
(5.1)\end{array}$ & $\begin{array}{c}63 \\
(19.2)\end{array}$ & $\begin{array}{c}76 \\
(22.8)\end{array}$ & $\begin{array}{c}89 \\
(26.7)\end{array}$ & $\begin{array}{c}55 \\
(16.5)\end{array}$ & $\begin{array}{c}23 \\
(6.9)\end{array}$ & $\begin{array}{c}5 \\
(1.5)\end{array}$ & $\begin{array}{c}4 \\
(1.2)\end{array}$ \\
\hline \multirow[t]{2}{*}{ Gender } & Male $(n=135)$ & $\begin{array}{c}6 \\
(4.4)\end{array}$ & $\begin{array}{c}25 \\
(18.5)\end{array}$ & $\begin{array}{c}36 \\
(26.6)\end{array}$ & $\begin{array}{c}31 \\
(23.0)\end{array}$ & $\begin{array}{c}29 \\
(21.5)\end{array}$ & $\begin{array}{c}6 \\
(4.5)\end{array}$ & $\begin{array}{c}1 \\
(0.7)\end{array}$ & $\begin{array}{c}1 \\
(0.7)\end{array}$ \\
\hline & Female $(n=197)$ & $\begin{array}{c}11 \\
(5.5)\end{array}$ & $\begin{array}{c}38 \\
(19.2)\end{array}$ & $\begin{array}{c}40 \\
(20.2)\end{array}$ & $\begin{array}{c}58 \\
(29.3)\end{array}$ & $\begin{array}{c}26 \\
(13.1)\end{array}$ & $\begin{array}{c}17 \\
(8.6)\end{array}$ & $\begin{array}{c}4 \\
(2.0)\end{array}$ & $\begin{array}{c}3 \\
(1.5)\end{array}$ \\
\hline \multirow[t]{2}{*}{ Dentition } & Mixed $(n=198)$ & $\begin{array}{c}9 \\
(4.5)\end{array}$ & $\begin{array}{c}29 \\
(14.6)^{*}\end{array}$ & $\begin{array}{c}48 \\
(24.2)\end{array}$ & $\begin{array}{c}52 \\
(26.3)\end{array}$ & $\begin{array}{c}37 \\
(18.7)\end{array}$ & $\begin{array}{l}15 \\
(7.6)\end{array}$ & $\begin{array}{c}4 \\
(2.0)\end{array}$ & $\begin{array}{c}4 \\
(2.0)\end{array}$ \\
\hline & Permanent $(n=134)$ & $\begin{array}{c}8 \\
(6.0)\end{array}$ & $\begin{array}{c}34 \\
(25.4)^{*}\end{array}$ & $\begin{array}{c}28 \\
(20.9)\end{array}$ & $\begin{array}{c}37 \\
(27.6)\end{array}$ & $\begin{array}{c}18 \\
(13.4)\end{array}$ & $\begin{array}{c}8 \\
(6.0)\end{array}$ & $\begin{array}{c}1 \\
(0.7)\end{array}$ & $\begin{array}{c}0 \\
(0.0)\end{array}$ \\
\hline \multirow[t]{8}{*}{ Occlusion } & Malocclusion $(n=214)$ & 25 & 87 & 116 & 128 & 85 & 31 & 7 & 8 \\
\hline & Increased overiet $(n=123)$ & $\begin{array}{c}8 \\
(6.5)\end{array}$ & $\begin{array}{c}17 \\
(13.8)\end{array}$ & $\begin{array}{c}27 \\
(22.0)\end{array}$ & $\begin{array}{c}33 \\
(26.8)\end{array}$ & $\begin{array}{c}27 \\
(22.0)\end{array}$ & $\begin{array}{c}7 \\
(5.7)\end{array}$ & $\begin{array}{c}1 \\
(0.8)\end{array}$ & $\begin{array}{c}3 \\
(2.4)\end{array}$ \\
\hline & Posterior crossbite $(n=50)$ & $\begin{array}{c}2 \\
(4.0)\end{array}$ & $\begin{array}{c}10 \\
(20.0)\end{array}$ & $\begin{array}{c}9 \\
(18.0)\end{array}$ & $\begin{array}{c}16 \\
132.0\end{array}$ & $\begin{array}{c}9 \\
(18.0)\end{array}$ & $\begin{array}{c}3 \\
(6.0)\end{array}$ & $\begin{array}{c}1 \\
(2.0)\end{array}$ & $\begin{array}{c}0 \\
(0.0)\end{array}$ \\
\hline & Frontal open bite $(n=24)$ & $\stackrel{1}{(4.2)}$ & $\begin{array}{c}2 \\
(8.3\end{array}$ & $\begin{array}{c}7 \\
(29.2)\end{array}$ & $\begin{array}{c}5 \\
(20.8)\end{array}$ & $\begin{array}{c}2 \\
(8.3)\end{array}$ & $\begin{array}{c}4 \\
(16.6)\end{array}$ & $\begin{array}{l}1 \\
(4.2)\end{array}$ & $\begin{array}{c}2 \\
(8.3)\end{array}$ \\
\hline & Deep overbite $(n=67)$ & $\begin{array}{c}1 \\
(1.5)\end{array}$ & $\begin{array}{c}14 \\
(20.1)\end{array}$ & $\begin{array}{c}19 \\
(28.4)\end{array}$ & $\begin{array}{c}20 \\
(29.8)\end{array}$ & $\begin{array}{c}12 \\
(17.9)\end{array}$ & $\begin{array}{c}1 \\
(1.5)\end{array}$ & $\begin{array}{c}0 \\
(0.0)\end{array}$ & $\begin{array}{c}0 \\
(0.0)\end{array}$ \\
\hline & Class II $(n=81)$ & $\begin{array}{c}6 \\
(7.4)\end{array}$ & $\begin{array}{c}12 \\
(14.8)\end{array}$ & $\begin{array}{c}22 \\
(27.2)\end{array}$ & $\begin{array}{c}15 \\
(18.5)\end{array}$ & $\begin{array}{c}16 \\
(19.7)\end{array}$ & $\begin{array}{c}6 \\
(7.4)\end{array}$ & $\begin{array}{c}2 \\
(2.5)\end{array}$ & $\begin{array}{c}2 \\
(2.5)\end{array}$ \\
\hline & Class III $(n=24)$ & $\begin{array}{c}1 \\
(4.2)\end{array}$ & $\begin{array}{c}6 \\
(25.0)\end{array}$ & $\begin{array}{c}5 \\
(20.8)\end{array}$ & $\begin{array}{c}6 \\
(25.0)\end{array}$ & $\begin{array}{c}4 \\
(16.6)\end{array}$ & $\begin{array}{c}2 \\
(8.3)\end{array}$ & $\begin{array}{c}0 \\
(0.0)\end{array}$ & $\begin{array}{c}0 \\
(0.0)\end{array}$ \\
\hline & Normal $(n=118)$ & $\begin{array}{c}6 \\
(5.1\end{array}$ & $\begin{array}{c}26 \\
(22.0)\end{array}$ & $\begin{array}{c}27 \\
(22.9)\end{array}$ & $\begin{array}{c}33 \\
(28.0)\end{array}$ & $\begin{array}{c}15 \\
(12.7)\end{array}$ & $\begin{array}{c}8 \\
(6.8)\end{array}$ & $\begin{array}{c}2 \\
(1.7)\end{array}$ & $\begin{array}{c}1 \\
(0.8)\end{array}$ \\
\hline
\end{tabular}

\footnotetext{
* Statistically different according to the Chi-squared test.
} 
The sample distribution and frequency of NOT-S domains, according the analyzed variables, are shown in Table 2 and Figure 1. The most frequent interview and examination domains demonstrated by the total sample were III (Habits) and IV (Chewing and Swallowing), with a frequency of 70.3 and $50.5 \%$, respectively, followed by domains 1 (Face at Rest), 3 (Facial Expression) and VI (Dryness of the mouth), with a frequency of 29.72, 27.62 and $24.32 \%$, respectively. Analyzing each domain between groups, gender and dentition, only domain 1 (Face at Rest) showed proportions significantly higher in the mixed dentition than the permanent dentition group, indicating that children with mixed dentition had more dysfunction in this domain. Almost $43 \%$ of children presented two or more habits. From these, $63.2 \%$ displayed onychophagy, $33.3 \%$ lip sucking or lip biting, $20.6 \%$ biting teeth together hard or grinding teeth during the day and $9.8 \%$ had sucking habits.

Table 2. Sample distribution and frequency (\%) of NOT-S domains

\begin{tabular}{|c|c|c|c|c|c|c|c|c|c|c|c|c|c|}
\hline & \multirow[b]{2}{*}{ Domain } & \multicolumn{6}{|c|}{ Interview } & \multicolumn{6}{|c|}{ Examination } \\
\hline & & 1 & II & III & IV & V & VI & 1 & 2 & 3 & 4 & 5 & 6 \\
\hline Total & & $\begin{array}{c}43 \\
(12.9 \%)\end{array}$ & $\begin{array}{c}31 \\
(9.3 \%)\end{array}$ & $\begin{array}{c}234 \\
(70.3 \%)\end{array}$ & $\begin{array}{c}168 \\
(50.5 \%)\end{array}$ & $\begin{array}{c}42 \\
(12.6 \%)\end{array}$ & $\begin{array}{c}81 \\
(24.3 \%)\end{array}$ & $\begin{array}{c}99 \\
(29.7 \%)\end{array}$ & $\begin{array}{c}14 \\
(4.2 \%)\end{array}$ & $\begin{array}{c}92 \\
(27.6 \%)\end{array}$ & $\begin{array}{c}40 \\
(12.0 \%)\end{array}$ & $\begin{array}{c}11 \\
(3.3 \%)\end{array}$ & $\begin{array}{c}20 \\
(6.3 \%)\end{array}$ \\
\hline \multirow[t]{2}{*}{ Gender } & $\begin{array}{l}\text { Male } \\
(n=135)\end{array}$ & $\begin{array}{c}12 \\
(8.9 \%)\end{array}$ & $\begin{array}{c}14 \\
(10.4 \%)\end{array}$ & $\begin{array}{c}86 \\
(63.7 \%)\end{array}$ & $\begin{array}{c}69 \\
(51.1 \%)\end{array}$ & $\begin{array}{c}19 \\
(14.1 \%)\end{array}$ & $\begin{array}{c}34 \\
(25.2 \%)\end{array}$ & $\begin{array}{c}45 \\
(33.3 \%)\end{array}$ & $\begin{array}{c}7 \\
(5.2 \%)\end{array}$ & $\begin{array}{c}35 \\
(25.9 \%)\end{array}$ & $\begin{array}{c}15 \\
(11.1 \%)\end{array}$ & $\begin{array}{c}4 \\
(2.9 \%)\end{array}$ & $\begin{array}{c}9 \\
(6.6 \%)\end{array}$ \\
\hline & $\begin{array}{l}\text { Female } \\
(n=197)\end{array}$ & $\begin{array}{c}31 \\
(15.7 \%)\end{array}$ & $\begin{array}{c}17 \\
(8.6 \%)\end{array}$ & $\begin{array}{c}148 \\
(75.1 \%)\end{array}$ & $\begin{array}{c}99 \\
(50.2 \%)\end{array}$ & $\begin{array}{c}23 \\
(11.7 \%)\end{array}$ & $\begin{array}{c}47 \\
(23.8 \%)\end{array}$ & $\begin{array}{c}54 \\
(27.4 \%)\end{array}$ & $\begin{array}{c}7 \\
(3.5 \%)\end{array}$ & $\begin{array}{c}57 \\
(28.9 \%)\end{array}$ & $\begin{array}{c}25 \\
(12.7 \%)\end{array}$ & $\begin{array}{c}7 \\
(3.5 \%)\end{array}$ & $\begin{array}{c}11 \\
(5.5 \%)\end{array}$ \\
\hline \multirow[t]{2}{*}{ Dentition } & $\begin{array}{l}\text { Mixed } \\
(n=198)\end{array}$ & $\begin{array}{c}28 \\
(14.1 \%)\end{array}$ & $\begin{array}{c}18 \\
(9.1 \%)\end{array}$ & $\begin{array}{c}138 \\
(69.7 \%)\end{array}$ & $\begin{array}{c}109 \\
(55.0 \%)\end{array}$ & $\begin{array}{c}30 \\
(15.1 \%)\end{array}$ & $\begin{array}{c}45 \\
(22.7 \%)\end{array}$ & $\begin{array}{c}74^{*} \\
(37.4 \%)\end{array}$ & $\begin{array}{c}6 \\
(3.0 \%)\end{array}$ & $\begin{array}{c}58 \\
(29.3 \%)\end{array}$ & $\begin{array}{c}28 \\
(14.1 \%)\end{array}$ & $\begin{array}{c}6 \\
(3.0 \%)\end{array}$ & $\begin{array}{c}16 \\
(8.1 \%)\end{array}$ \\
\hline & $\begin{array}{l}\text { Permanent } \\
(n=134)\end{array}$ & $\begin{array}{c}15 \\
(11.2 \%)\end{array}$ & $\begin{array}{c}13 \\
(9.7 \%)\end{array}$ & $\begin{array}{c}96 \\
(71.6 \%)\end{array}$ & $\begin{array}{c}59 \\
(44.0 \%)\end{array}$ & $\begin{array}{c}12 \\
(8.9 \%)\end{array}$ & $\begin{array}{c}36 \\
(26.9 \%)\end{array}$ & $\begin{array}{c}25^{*} \\
(18.6 \%)\end{array}$ & $\begin{array}{c}8 \\
(6.0 \%)\end{array}$ & $\begin{array}{c}34 \\
(25.4 \%)\end{array}$ & $\begin{array}{c}12 \\
(8.9 \%)\end{array}$ & $\begin{array}{c}5 \\
(3.7 \%)\end{array}$ & $\begin{array}{c}4 \\
(3.0 \%)\end{array}$ \\
\hline \multicolumn{14}{|c|}{ Malocclusion $(n=214)$} \\
\hline & $\begin{array}{l}\text { Increased } \\
\text { overjet } \\
(n=123)\end{array}$ & $\begin{array}{c}13 \\
(10.6 \%)\end{array}$ & $\begin{array}{c}13 \\
(10.6 \%)\end{array}$ & $\begin{array}{c}85 \\
(69.1 \%)\end{array}$ & $\begin{array}{c}59 \\
(48.0 \%)\end{array}$ & $\begin{array}{c}19 \\
(15.4 \%)\end{array}$ & $\begin{array}{c}27 \\
(21.9 \%)\end{array}$ & $\begin{array}{c}40 \\
(35.5 \%)\end{array}$ & $\begin{array}{c}2 \\
(1.6 \%)\end{array}$ & $\begin{array}{c}42 \\
(34.1 \%)\end{array}$ & $\begin{array}{c}10 \\
(8.1 \%)\end{array}$ & $\begin{array}{c}3 \\
(2.4 \%)\end{array}$ & $\begin{array}{c}7 \\
(5.7 \%)\end{array}$ \\
\hline & $\begin{array}{l}\text { Posterior } \\
\text { crossbite } \\
(n=50)\end{array}$ & $\begin{array}{c}8 \\
(16.0 \%)\end{array}$ & $\begin{array}{c}4 \\
(8.0 \%)\end{array}$ & $\begin{array}{c}36 \\
(72.0 \%)\end{array}$ & $\begin{array}{c}27 \\
(54.0 \%)\end{array}$ & $\begin{array}{c}4 \\
(8.0 \%)\end{array}$ & $\begin{array}{c}11 \\
(22.0 \%)\end{array}$ & $\begin{array}{c}15 \\
(30.0 \%)\end{array}$ & $\begin{array}{c}2 \\
(4.0 \%)\end{array}$ & $\begin{array}{c}16 \\
(32.0 \%)\end{array}$ & $\begin{array}{c}8 \\
(16.0 \%)\end{array}$ & $\begin{array}{c}0 \\
(0.0 \%)\end{array}$ & $\begin{array}{c}2 \\
(4.0 \%)\end{array}$ \\
\hline & $\begin{array}{l}\text { Frontal } \\
\text { open bite } \\
(n=24)\end{array}$ & $\begin{array}{c}5 \\
(20.8 \%)\end{array}$ & $\begin{array}{c}5 \\
(20.8 \%)\end{array}$ & $\begin{array}{c}14 \\
(58.3 \%)\end{array}$ & $\begin{array}{c}12 \\
(50.0 \%)\end{array}$ & $\begin{array}{c}8^{*} \\
(33.3 \%)\end{array}$ & $\begin{array}{c}6 \\
(25.0 \%)\end{array}$ & $\begin{array}{c}11 \\
(45.8 \%)\end{array}$ & $\begin{array}{c}1 \\
(4.2 \%)\end{array}$ & $\begin{array}{c}7 \\
(29.2 \%)\end{array}$ & $\begin{array}{c}5 \\
(20.8 \%)\end{array}$ & $\begin{array}{c}0 \\
(0.0 \%)\end{array}$ & $\begin{array}{c}5^{*} \\
(20.8 \%)\end{array}$ \\
\hline & $\begin{array}{l}\text { Deep } \\
\text { overbite } \\
(n=67)\end{array}$ & $\begin{array}{c}7 \\
(10.4 \%)\end{array}$ & $\begin{array}{c}5 \\
(7.5 \%)\end{array}$ & $\begin{array}{c}47 \\
(70.1 \%)\end{array}$ & $\begin{array}{c}34 \\
(50.7 \%)\end{array}$ & $\begin{array}{c}4 \\
(6.0 \%)\end{array}$ & $\begin{array}{c}12 \\
(17.9 \%)\end{array}$ & $\begin{array}{c}20 \\
(29.8 \%)\end{array}$ & $\begin{array}{c}3 \\
(4.5 \%)\end{array}$ & $\begin{array}{c}21 \\
(31.3 \%)\end{array}$ & $\begin{array}{c}6 \\
(8.9 \%)\end{array}$ & $\begin{array}{c}3 \\
(4.5 \%)\end{array}$ & $\begin{array}{c}3 \\
(4.5 \%)\end{array}$ \\
\hline & $\begin{array}{l}\text { Class II } \\
(n=81)\end{array}$ & $\begin{array}{c}10 \\
(12.34 \%)\end{array}$ & $\begin{array}{c}6 \\
(7.4 \%)\end{array}$ & $\begin{array}{c}60 \\
(74 \%)\end{array}$ & $\begin{array}{c}40 \\
(49.4 \%)\end{array}$ & $\begin{array}{c}12 \\
(14.8 \%)\end{array}$ & $\begin{array}{c}19 \\
(23.5 \%)\end{array}$ & $\begin{array}{c}28 \\
(34.6 \%)\end{array}$ & $\begin{array}{c}5 \\
(6.2 \%)\end{array}$ & $\begin{array}{c}22 \\
(27.16 \%)\end{array}$ & $\begin{array}{c}10 \\
(12.3 \%)\end{array}$ & $\begin{array}{c}2 \\
(2.5 \%)\end{array}$ & $\begin{array}{c}7 \\
(8.65 \%)\end{array}$ \\
\hline & $\begin{array}{l}\text { Class III } \\
(n=24)\end{array}$ & $\begin{array}{c}7 \\
(29.2 \%)\end{array}$ & $\begin{array}{c}2 \\
(8.3 \%)\end{array}$ & $\begin{array}{c}17 \\
(70.8 \%)\end{array}$ & $\begin{array}{c}13 \\
(54.2 \%)\end{array}$ & $\begin{array}{c}1 \\
(4.2 \%)\end{array}$ & $\begin{array}{c}5 \\
(20.8 \%)\end{array}$ & $\begin{array}{c}6 \\
(25.0 \%)\end{array}$ & $\begin{array}{c}1 \\
(4.2 \%)\end{array}$ & $\begin{array}{c}6 \\
(25.0 \%)\end{array}$ & $\begin{array}{c}2 \\
(8.3 \%)\end{array}$ & $\begin{array}{c}0 \\
(0.0 \%)\end{array}$ & $\begin{array}{c}0 \\
(0.0 \%)\end{array}$ \\
\hline \multicolumn{2}{|c|}{$\begin{array}{l}\text { Normal Occlusion } \\
(n=118)\end{array}$} & $\begin{array}{c}14 \\
(11.9 \%)\end{array}$ & $\begin{array}{c}13 \\
(11.0 \%)\end{array}$ & $\begin{array}{c}80 \\
(67.8 \%)\end{array}$ & $\begin{array}{c}62 \\
(52.5 \%)\end{array}$ & $\begin{array}{c}14 \\
(11.9 \%)\end{array}$ & $\begin{array}{c}30 \\
(25.4 \%)\end{array}$ & $\begin{array}{c}27 \\
(22.9 \%)\end{array}$ & $\begin{array}{c}7 \\
(5.9 \%)\end{array}$ & $\begin{array}{c}24 \\
(20.3 \%)\end{array}$ & $\begin{array}{c}14 \\
(11.9 \%)\end{array}$ & $\begin{array}{c}7 \\
(5.9 \%)\end{array}$ & $\begin{array}{c}6 \\
(5.1 \%)\end{array}$ \\
\hline
\end{tabular}

* Statistically different according to the Chi-squared test.

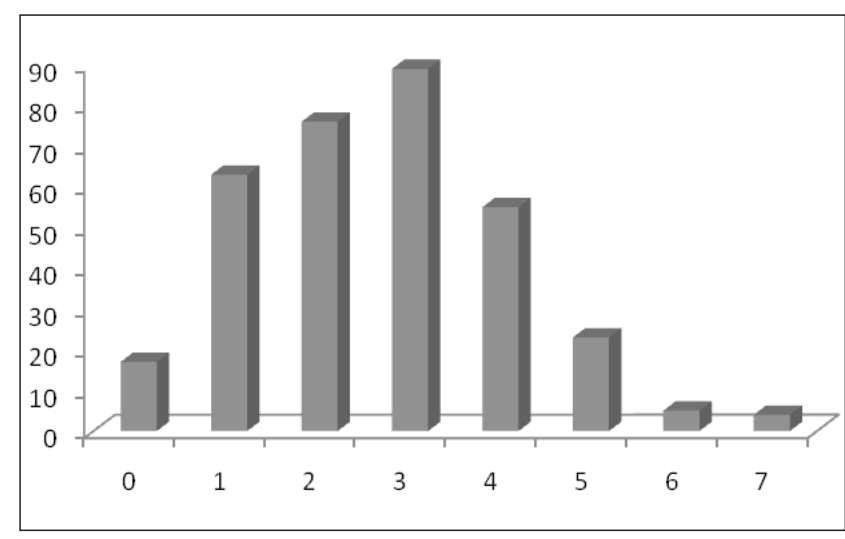

Fig. 1. Distribution of the NOT-S scores

\section{NOT-S validity and reliability}

The mean NOT-S score was 2.64, and the differences between groups, considering gender, dentition and type of occlusion, are shown in Table 3 . The ability of the protocol to discriminate between genders was not confirmed, due to the similarity of male and female means $(P>0.05)$. However, the test discriminated significantly between dentitions and occlusions. The children in the mixed dentition group showed higher means than those with permanent dentition, as well as children with frontal open bite in relation to children with normal occlusion. Upon reapplication of the protocol (testretest), only $2.19 \%$ of answers or assessed sentences were different; there was an agreement of $97.81 \%$ comparing the first and retest evaluations. The Kappa value of each domain is shown in Table 4. 
Table 3. Discriminant validity of the NOT-S scores according to gender, type of dentition and occlusion

\begin{tabular}{lcccc}
\hline \multicolumn{1}{c}{ Groups } & Number & Mean & SD & $P$-value \\
\hline $\begin{array}{l}\text { Total } \\
\text { Gender }\end{array}$ & 332 & 2.64 & 1.45 & - \\
$\quad$ Male & 135 & 2.59 & 1.35 & 0.38 \\
$\quad$ Female & 197 & 2.67 & 1.51 & \\
Dentition & & & & \\
Mixed & 198 & 2.81 & $1.49^{*}$ & 0.007 \\
Permanent & 134 & 2.38 & $1.35^{*}$ & \\
Occlusion & 123 & 2.76 & 1.49 & 0.08 \\
Increased overiet & 50 & 2.57 & 1.36 & 0.23 \\
Posteriorcrossbite & 24 & 3.29 & $1.88^{* *}$ & 0.04 \\
Frontal open bite & 67 & 2.46 & 1.11 & 0.49 \\
Deep overbite & 81 & 2.73 & 1.61 & 0.20 \\
Class II & 24 & 2.50 & 1.38 & 0.496 \\
Class III & 118 & 2.53 & $1.43^{* *}$ & \\
Normal & & & & \\
\hline
\end{tabular}

* $P<0.01$, intra-group comparison using the Mann-Whitney $U$ test.

** $P<0.05$, comparisons between each malocclusion type and normal occlusion

SD: standard deviations.

\begin{tabular}{lcl}
\hline \multicolumn{1}{c}{ Orofacial dysfunction } & Statistical tests & \multicolumn{1}{c}{ Interpretation } \\
\cline { 2 - 3 } & Kappa value & \multicolumn{1}{c}{ Kappa } \\
\hline NOT-S interview & & \\
I - Sensory function & 0.80 & Substantial agreement \\
II - Breathing & 0.96 & Almost perfect agreement \\
III - Habits & 0.68 & Substantial agreement \\
IV - Chewing and swallowing & 0.76 & Substantial agreement \\
V - Drooling & 0.88 & Almost perfect agreement \\
VI - Dryness of the mouth & 0.26 & Fair agreement \\
NOT-S examination & & \\
1 - Face at rest & 0.52 & Moderate agreement \\
2 - Nose breathing & 0.96 & Almost perfect agreement \\
3 - Facial expression & 0.20 & Slight agreement \\
4 - Masticatory muscle and jaw function & 0.84 & Almost perfect agreement \\
5 - Oral motor function & 0.94 & Almost perfect agreement \\
6 - Speech & 0.94 & Almost perfect agreement \\
\hline
\end{tabular}

Table 4 . Reliability and reproducibility of NOT-S, evaluated by test-retest of 50 patients and validated for children aged 6 to 12 years, differing from NOT-S, which can be applied to different age groups and is more simple and rapid to administer (1,11-13). Both procedures permit the examiner to numerically express his perception of the characteristics and behaviors observed (i.e., they permit the measurement of the impressions of the clinician).

During the application of NOT-S, we observed a frequency of $5 \%$ for a score of 0 , meaning either that most children presented some impairment or some false positive results were recorded. Bakke et al. (1) observed that the sensitivity of the NOT-S was high, but the specificity was slightly less than desired. Indeed, $63 \%$ of the health group presented a score of 0 , indicating a risk for false positives. 
Nevertheless, these authors considered that a few false positive results are preferable to a few false negatives (1). Moreover, our subjects aged from 8 to 14 years. The focus on this specific age group, consisting of children, and the domains most frequently affected, suggests normative data of the assessed sample. In this context, our findings are in line with Stahl et al. (3), who found a low percentage $(10.2 \%)$ of children in mixed dentition (mean age 8.3 years) without functional disorders (mouth posture, tongue posture, swallowing patterns and articulation - production of sounds) or oral habits, using their own protocol.

For the total sample, the mean score was 2.64 (range $0-7)$. There was no difference between genders, but children with mixed dentition showed higher values than those with permanent dentition. This finding may be justified by the fact that mixed dentition is a transitory period, as observed by Stahl et al. (3), who verified that every third child in a mixed dentition group presented three or more dysfunctions. In a sample of healthy children (from 3 to 6 years old) examined with NOT-S, Gustavsson et al. ${ }^{12}$ found a total mean score of $1.38 \pm 0.97$, without significant differences regarding age or gender. However, NOT-S scores decreased with increasing age. These results and the higher values found in the mixed dentition group could suggest that maturation of oral functions may improve the respective performance in children. This is in agreement with Finan and Smith (14), who considered that the oral sensorimotor pathway matures throughout childhood in concert with the continued acquisition of complex motor skills.

In relation to occlusal findings, the normal occlusion group represented $35.5 \%$ of the studied population, a value previously observed by Emerich and Wojtaszek-Slominska (15). The frontal open bite was the only occlusal finding that had an influence on NOT-S scores. This is due to the higher values compared to the normal occlusion group, likely because this malocclusion is a consequence of the presence of habits and results in deviation of lip position and impairment of nose breathing. However, Grabowski et al. (16) determined that four types of malocclusion (frontal open bite, lateral crossbite, reduced overjet and increased overjet) were more significant functional disturbances on comparing the respective frequencies, thus differing from the present study in which the mean scores were compared among malocclusion groups.

In the interview, we observed that domain III (Habits) and domain IV (Chewing and Swallowing) had a frequency of 70 and $50 \%$, respectively. These findings demonstrate that the majority of children in the current study had some type of habit (the most frequent one was nail biting, 63.2\%) and difficulty in eating hard food (the most related problem in the Chewing and Swallowing domain), and these were the main influencing factors on orofacial dysfunction. For Bakke et al. (1), the highest frequency of interview domains, considering the data of healthy subjects, were domains II and III (11.7\%), whereas for the clinically referred sample, domain IV was the most prevalent $(63.3 \%)$. The latter was also observed by Bergendal et al. (11) in patients with ectodermal dysplasia (82.6\%). In the present study, we applied the NOT-S to randomly selected subjects within a specific age group, whereas Bakke et al. (1) and Bergendal et al. (11) performed the screening with NOT-S in patients with different kinds of orofacial impairments and in a large age range. Bakke et al. (1) examined subjects with neoplasms, mental disorders, diseases of the digestive system and other diseases of the International Classification of Diseases (ICD10) classifications (17).

These facts alerted us to the possibility of a high level of anxiety in these children, which is suggested by the literature (i.e., nail biting demonstrates anxiety that is made worse by tense moments, as a reflex of emotional imbalances (18). Moreover, the eating habits of families in Brazil who consume unhealthy and industrialized food (soft drinks, fried snacks, potato chips, sandwiches, snacks package, biscuits/cookies and candies/sweets/chocolates) with a high frequency (19) could be influencing the results related to domain IV. These considerations suggest that further studies should be carried out, taking in account these variables.

No examination domain showed differences between genders and malocclusions, except domain 1 (Face at Rest), in which there was a statistically significant difference between the mixed and permanent dentition groups. The most assessed dysfunction in this domain was "deviant lip position". This suggests a reduction of this dysfunction from mixed to permanent dentition.

In principle, when a point is scored in one domain in the screening, a more detailed evaluation of that domain, and if necessary, a referral to a specialist may be considered. Indeed, Bakke et al. (1) suggested that scores equal to or below 2 points should be carefully considered before a referral (1). However, the present and the Gustavsson et al. (12) studies are the unique ones that evaluated orofacial dysfunction using NOT-S on a random sample of healthy children, as previously recommended (1). Further studies are still needed icluding other disabilities and ages to improve the overall estimates of changes in orofacial function over time and/or rough assessments of treatment effects (1), as done by Lundeborg et al. (13). They verified that tonsillectomy in preschool children results in an oral motor function improvement, with a similar degree in relation to controls, as measured by NOT-S.

It is very important to verify the reproducibility (test re-test reliability) because every assessment protocol must be reproducible over time (i.e., must produce equal or very similar results in two or more assessments of the same patient) as long as the clinical state of the patient did not change (20). The intra-examiner reproducibility was demonstrated, and the rate obtained (97.8\% agreement) was very close to that found by Bakke et al. (1) (95-99\%). All of the domains had good agreement according to their kappa value, with the exception of the domains VI (dryness of the mouth) and 3 (facial expression), which had fair and slight agreement, respectively. This is likely because these variables could be suffering from different stimuli during each examination. 


\section{Conclusions}

In conclusion, the Brazilian version of NOT-S was considered into validated, making possible its use and comparison with international data. Our findings showed no differences in NOT-S scores between genders, but subjects in mixed dentition and those with frontal open bite presented more orofacial dysfunction. "Habits" and "Chewing and Swallowing" were the most affected domains (III and IV, respectively).

\section{Acknowledgements}

The authors gratefully acknowledge Lourival Antonio Mesquita, Marinês Nobre dos Santos Uchôa and Renata de Oliveira Mattos Graner for participation in translation process. Financial support from the State of São Paulo Research Foundation (FAPESP, SP, Brazil, n. 2007/ 06863-0), the volunteers and their parents are also greatly acknowledged. of a comprehensive screening for orofacial dysfunction. Swed Dent J 2007;31:75-84.

2. Grabowski R, Stahl F, Gaebel M, Kundt G. Relationship between occlusal findings and orofacial myofunctional status in primary and mixed dentition. Part I: Prevalence of malocclusions. J Orofac Orthop 2007;68:26-37.

3. Stahl F, Grabowski R, Gaebel M, Kundt G. Relationship between occlusal findings and orofacial myofunctional status in primary and mixed dentition. Part II: Prevalence of orofacial dysfunctions. J Orofac Orthop 2007;68:74-90.

4. Lund JP. Mastication and its control by the brain stem. Crit Rev Oral Biol Med 1991;2:33 64.

5. John MT, Hirsch C, Reiber T, Dworkin S. Translating the research diagnostic criteria for temporomandibular disorders into German: evaluation of content and process. J Orofac Pain 2006;20:43-52.

6. Barbosa TS, Tureli MCM, Gavião MB. Validity and reliability of the Child Perceptions Questionnaires applied in Brazilian children. BMC Oral Health 2009;9:13.

7. Leme MS, Barbosa Ts, Gavião MB. [Brazilian version of the Nordic Orofacial Test Screening (NOT-S) for evaluation of orofacial dysfunctions]. Pesq Bras Odontoped Clin Integr $2011 ; 11: 281-9$.

8. Guillemin F, Bombardier C, Beaton D. Cross-cultural adaptation of health-related quality of life measures: literature review and proposed guidelines. J Clin Epidemiol 1993;46(12): 1417-32.

9. World Health Organization. Oral health surveys. Basic methods. Geneva: World Health Organization; 1997.

10. Felício CM, Ferreira CL. Protocol of orofacial myofunctional evaluation with scores. Int J Pediatr Otorhinolaryngol 2008;72:367-75.

11. Bergendal B, McAllister A, Stecksen-Blicks C. Orofacial dysfunction in ectodermal dysplasias measured using the Nordic Orofacial Test-Screening protocol. Acta Odontol Scand. 2009: 1-5.

12. Gustavsson C, Skoglund C, Thelin H. Norm data for the Nordic Orofacial Test-Screening (NOTS) for children aged 3 to 6 years. [Accessed on $2011 \mathrm{Jul}$ 28]. Available at: http:// liu.diva-portal.org/smash/record.jsf? pid=diva2:239055

13. Lundeborg I, McAllister A, Graf J, Ericsson E, Hultcrantz E. Oral motor dysfunction in children with adenotonsillar hypertrophy--effects of surgery. Logoped Phoniatr Vocol $2009 ; 34: 111-6$.

14. Finan DS, Smith A. Jaw stretch reflexes in children. Exp Brain Res 2005; 164:58-66.

15. Emerich K, Wojtaszek-Slominska A. Clinical practice. Later orthodontic complications caused by risk factors observed in the early years of life. Eur J Pediatr 2010;169:651-5.

16. Grabowski R, Kundt G, Stahl F. Interrelation between occlusal findings and orofacial myofunctional status in primary and mixed dentition: Part III: Interrelation between malocclusions and orofacial dysfunctions. J Orofac Orthop 2007;68:462-76.

17. ICD-10. International statistical classification of diseases and related health problems. Tenth revision. Geneva: World Health Organization; 1993.

18. Tanaka OM, Vitral RW, Tanaka GY, Guerrero AP, Camargo ES. Nailbiting, or onychophagia: a special habit. Am J Orthod Dentofacial Orthop 2008;134:305-8.

19. Marchioni DM, Claro RM, Levy RB, Monteiro CA. Patterns of food acquisition in Brazilian households and associated factors: a population-based survey. Public Health Nutr $2011 ; 14: 1586-92$.

20. Ware JE, Gandek B. IQOLA Project Group: The SF-36 health survey: development and use in mental health research and the IQOLA project. Int J Ment Health 1994;23:49-73. 